\title{
LE BILAN DU «FÉDÉRALISME D'OUVERTURE» DU GOUVERNEMENT DE STEPHEN HARPER FACE AUX REVENDICATION DU QUÉBEC
}

Gregory Godbout

Depuis la signature de l'Acte de l'Amérique du Nord britannique, qui marque la formation de la fédération canadienne en 1867, se sont alternées des périodes de centralisation du pouvoir entre les mains du palier fédéral et d'autres où les provinces arrivaient à faire quelques gains. ${ }^{1}$ Les relations entre le gouvernement fédéral et les provinces, surtout celles avec le Québec, sont souvent tendues. Nous n'avons qu'à penser à la naissance du mouvement souverainiste québécois et à ses revendications. Pierre-Elliott Trudeau, premier ministre canadien de l'époque, s'opposait fermement au mouvement souverainiste québécois ${ }^{2}$, lui qui était un fervent défenseur des droits individuels, dont la pensée est illustrée par des réformes comme la loi sur le multiculturalisme, la loi sur le bilinguisme et le rapatriement constitutionnel qui y incluait la Charte canadienne des droits et libertés ${ }^{3}$. Même si ces mesures avaient pour but l'unification nationale par la création d'une identité canadienne basée sur des valeurs communes mesures ont «[...]laissé le pays plus divisé que jamais» selon Kenneth McRoberts, car cette vision du Canada basée sur l'égalité des individus et des provinces allait à l'encontre des revendications collectives des Québécois ${ }^{4}$.

La décennie entre la victoire du «non » lors du référendum sur la souveraineté en 1995

\footnotetext{
${ }^{1}$ R. Pelletier et M. Tremblay. Le parlementarisme canadien (Québec 2009), 59.

${ }^{2}$ D. Monière. Pour comprendre le nationalisme au Québec et ailleurs (Québec 2001), 117.

${ }^{3}$ C. Couture et D. Chandonnet. Vingt ans après Charlottetown : L'ordre libéral au Canada, Québec, Les Presses de l'Université Laval, 2013, p. 25-46.

${ }^{4}$ R. Pelletier et M. Tremblay. Le parlementarisme canadien (Québec 2009), 65.
} 
au Québec et l'élection de Stephen Harper à la tête du gouvernement canadien peut être qualifiée d'ère de confrontation entre le gouvernement fédéral, dirigé par le Parti Libéral du Canada, et la province du Québec ${ }^{5}$. Dès 2004, Stephen Harper s'engage à sortir de cette ère s'il prend le pouvoir et prône un « fédéralisme d'ouverture ». Une fois au pouvoir en 2006, il a redéfini sa doctrine en disant qu'une telle pratique représenterait une opportunité de libérer le Québec de la polarisation au sein de sa population par la mise en œuvre d'actions concrètes telles que le respect des compétences provinciales et la limitation du pouvoir de dépenser ${ }^{6}$. Son discours faisait donc la promesse d'un renouvellement de la dynamique des relations intergouvernementales ${ }^{7}$.

Les relations intergouvernementales entre le Canada et le Québec sont souvent sous les feux de la rampe dans les médias. Le dossier constitutionnel est au cœur de cet enjeu. Il est important de rappeler que les différents gouvernements québécois qui se sont succédé depuis le rapatriement constitutionnel de 1982 ont tous refusé de signer la Constitution dans son état actuel. Certains fédéralistes québécois croient que le Québec pourrait y donner son accord moyennant des amendements majeurs, nous n'avons qu'à penser aux cinq conditions minimales que proposaient le Premier ministre du Québec Robert Bourassa pour que le Québec accepte de signer la Constitution canadienne lors des accords du Lac Meech ${ }^{8}$. Quant à eux, bien des souverainistes considèrent que la constitution actuelle est la preuve que les intérêts du Québec ne

\footnotetext{
${ }^{5}$ R. Pelletier et M. Tremblay. Le parlementarisme canadien (Québec 2009), 73.

${ }^{6}$ A. Harmes. The Political Economy of Open Federalism (2007), 420.

${ }^{7}$ A. Noel. Fédéralisme d'ouverture et pouvoir de dépenser au Canada (2008), 1.

${ }^{8}$ E. Montpetit. Le fédéralisme d'ouverture : La recherche d'une légitimité canadienne au Québec (Québec 2007), 29.
} 
peuvent être protégés dans le cadre constitutionnel actuel et que cela justifie l'indépendance ${ }^{9}$. Il y a donc consensus entre les différents partis à l'Assemblée nationale, avec plusieurs distinctions bien sûr, que le fédéralisme canadien comme il a été institutionnalisé en 1982 ne concorde pas avec la vision que bien des Québécois se font du Canada ${ }^{10}$. Afin de bien saisir l'enjeu entourant notre article, il sera important de circonscrire le concept de fédération ainsi que son corollaire beaucoup plus spécifique qu'est le « fédéralisme d'ouverture » dans le contexte politique canadien.

Tout d'abord, le concept de fédération sera défini. Selon l'équipe de Perspective Monde de l'Université de Sherbrooke, une fédération est un pays où la constitution sépare les pouvoirs en au moins deux ordres politiques distincts ${ }^{11}$. Cela suppose donc que les compétences législatives sont réparties entre le pallier fédéral et les pouvoirs régionaux (provinces, cantons, états, républiques) ${ }^{12}$. Un État fédéral s'oppose à un État unitaire, ou seul le pouvoir central peut légiférer. Dans une fédération, le pouvoir central ne détient donc pas la pleine souveraineté car elle est partagée, selon divers degrés, avec les pouvoirs régionaux ${ }^{13}$. Un élément important de ce type de d'organisation de l'État, est la non-subordination des subdivisions au gouvernement central. En effet, Ottawa ne peut dissoudre les assemblées législatives provinciales et la légitimité de celles-ci émane de la relation que ces provinces entretiennent avec leurs populations respectives.

\footnotetext{
${ }^{9} \mathrm{G}$. Laforest. The 20th annual Mcdonald lecture in constitutional studies: What Canadian Federalism Means in Québec (2010), 3.

${ }^{10} \mathrm{M}$. Bock-Cöté. De la reconnaissance du Québec à celle des Québécois : retour sur la reconnaissance de la nation par le gouvernement de Stephen Harper à l'automne 2006 (2007), 6.

${ }^{11}$ Équipe Perspective Monde. Définition de Fédération dans le glossaire (2013).

${ }^{12}$ Ibid.

${ }^{13}$ R. Pelletier. La dynamique fédérale au Canada dans «Le fédéralisme en Belgique et au Canada (2009), 1.
} 
Philip Resnick distingue deux types de fédérations : Les fédérations territoriales et les fédérations multinationales. Au sein des premières est proposé une vision commune de la nationalité alors qu'au sein des secondes, les diverses nations qui la composent ont droit à une certaine reconnaissance $^{14}$. Selon lui, ces deux visions sont présentes au Canada, car la plupart des Québécois envisagent la fédération comme étant multinationale, tandis que la plupart des habitants des neuf autres provinces la considèrent surtout comme étant territoriale ${ }^{15}$. Cette division est clairement illustrée par les sondages qui démontrent que les résidents du Québec se définissent d'avantage comme Québécois que comme Canadiens et les résidents des autres provinces ont en général développé un sentiment d'appartenance beaucoup plus grand envers le Canada $^{16}$. En reconnaissant des droits collectifs aux peuples autochtones aux articles 25 et 35 de la Charte, la constitution canadienne présente certains aspects d'une fédération multinationale ${ }^{17}$. Alain Noël complète en affirmant que le Canada est de fait une fédération multinationale, par les différentes nations qui la composent, autant autochtones, québécoises ou acadiennes, mais fonctionne bien souvent comme si elle était territoriale ${ }^{18}$.

Réjean Pelletier rappelle à quel point les suites du rapatriement constitutionnel de 1982, c'est-à-dire les tentatives de réformes constitutionnelles, ont marqué l'imaginaire collectif canadien et font en sorte que le désir de rouvrir le dossier constitutionnel est très faible parmi les décideurs politiques et la population. ${ }^{19}$. Il rappelle aussi l'incompréhension de la plupart des habitants du Canada face aux revendications historiques du Québec et d'un manque de volonté

\footnotetext{
${ }^{14}$ P. Resnick. Le Canada : une fédération territoriale ou multinationale (2013), 21.

${ }^{15}$ Ibid., 29.

${ }^{16}$ Ibid., 34.

${ }^{17}$ R. Pelletier et M. Tremblay. Le parlementarisme canadien (Québec 2009), 78.

${ }^{18}$ A. Noel. Fédéralisme d'ouverture et pouvoir de dépenser au Canada (2008), 13.

${ }^{19}$ R. Pelletier. Le renouvellement de la fédération canadienne : Quand l'immobilisme tient lieu de politique (2013), 55.
} 
du côté canadien à faire des concessions ${ }^{20}$. À titre d'exemple, le Québec avait comme revendications la reconnaissance du Québec comme société distincte, l'octroi d'un droit de véto au Québec pour toutes modifications à la constitution, le droit de retrait avec compensation pour tout programme fédéral représentant une ingérence dans les compétences provinciales, plus de pouvoirs en matière d'immigration ainsi que la garantie que le Québec puisse sélectionner trois des neufs juges à la Cour suprême. Selon Éric Montpetit, il y a aurait un espace dans l'ordre constitutionnel actuel qui permettrait de venir apaiser l'inconfort québécois face au fédéralisme canadien et qui se définit autour de ces points : La reconnaissance du caractère dualiste du Canada, la légitimation de l'utilisation de la clause dérogatoire, le respect des compétences provinciales, l'attribution du pouvoir à l'Assemblée nationale de nommer les juges à la Cour suprême du Canada, le règlement du déséquilibre fiscal ainsi que l'encadrement du pouvoir de dépenser ${ }^{21}$.

Quant à lui, Adam Harmes vient ajouter une autre dimension au fédéralisme d'ouverture qui, selon lui, s'insère à merveille dans l'idéologie néolibérale dont est adepte le Parti conservateur $^{22}$. Il démontre qu'une telle décentralisation, en engendrant une réduction de la taille de l'État fédéral, engendrerait indirectement une semblable réduction au niveau provincial à cause de la compétition pour les taxes et les impôtts ${ }^{23}$.

Considérant qu'il y aurait un espace au sein de l'ordre constitutionnel canadien actuel permettant au gouvernement fédéral de calmer l'inconfort ressenti par une bonne partie de la population québécoise par rapport au fédéralisme canadien et cela sans procéder à une réforme

\footnotetext{
${ }^{20}$ Ibid., 51.

${ }^{21}$ E. Montpetit. Le fédéralisme d'ouverture (Québec, 2007), 62-78.

${ }^{22}$ A. Harmes. The Political Economy of Open Federalism (2007), 422.

${ }^{23}$ Ibid., 433.
} 
constitutionnelle, il sera intéressant de se demander où se situe Stephen Harper aujourd'hui face à cet engagement électoral. À ce jour, peu d'études ont été faites permettant de porter un tel constat. En passant en revue les aspects saillants des relations intergouvernementales entre le Canada et le Québec depuis son arrivée au pouvoir en 2006, quel bilan peut-on faire du « fédéralisme d'ouverture » de Stephen Harper?

Sans porter de jugement trop hâtif, j'estime que le bilan de son ouverture face aux revendications du Québec est assez mitigé. Malgré l'apparence d'ouverture et les certes quelques avancées, «[...] il importe de préciser que les résultats demeurent encore assez limités» ${ }^{24}$. Le bilan traitera principalement de deux grands aspects, premièrement des mesures qui tendent vers la reconnaissance de la dualité des peuples fondateurs du Canada, c'est-à-dire s'approchant d'une vision multinationale du fédéralisme et se distanciant donc de l'idéal du «Canada one nation» constitutionalisé lors du rapatriement de $1982^{25}$. La motion reconnaissant la nation québécoise ainsi que l'octroi d'un siège à l'Unesco pour le Québec seront traités.

Deuxièmement, il sera question du respect du partage des compétences. Plus spécifiquement, on se penchera sur le règlement du déséquilibre fiscal et l'utilisation du pouvoir de dépenser.

Tout d'abord, le gouvernement canadien de Stephen Harper a soumis à la Chambre des communes, en 2006, une motion reconnaissant les Québécois et les Québécoises comme formant une nation au sein d'un Canada uni ${ }^{26}$. Ce faisant, il reconnaissait la distinction québécoise, mais sans l'enchâssement constitutionnel qu'aurait permis la réussite des Accords constitutionnels

\footnotetext{
${ }^{24}$ R. Pelletier et M. Tremblay. Le parlementarisme canadien (Québec 2009), 74.

${ }^{25}$ M. Bock-Cöté. De la reconnaissance du Québec (2007), 10.

${ }^{26} \mathrm{R}$. Dutrisac et La Presse Canadienne. Le Québec reconnu comme nation-Une motion sans conséquence, avoue Ottawa (2006).
} 
manqués de Meech et de Charlottetown ${ }^{27}$.

Pour Philip Resnick, en reconnaissant les Québécois comme une nation et non le Québec la motion évitait de manière habile de reconnaître le caractère national du Québec et toute possibilité de lier l'identité nationale québécoise avec le pouvoir politique du Québec, ce qui est incompatible avec la vision mono-nationale du Canada, excluant bien-sûr la reconnaissance des droits collectifs des peuples autochtones et les différents traités signés avec ceux-ci ${ }^{28}$. Comme le rappelle Mathieu Bock-Côté, cette reconnaissance n'a pas eu d'effets politiques, ses effets se limitant à une portée purement symbolique ${ }^{29}$. Il l'interprète même comme étant une tentative de «[...]décrocher durablement le désir de reconnaissance identitaire et symbolique de la nation québécoise de ses conséquences politiques[... $]^{30}$.» Vision quelque peu pessimiste du geste de Stephen Harper, à moins que l'on considère ce geste comme purement électoraliste, visant à agréger les votes du plus de Québécois possible alors qu'il ne formait qu'un gouvernement minoritaire. Mathieu Bock-Côté rappelle que les principes l'orientant ne sont clairement pas un désir de redéfinir l'identité canadienne sur sa matrice dualiste ${ }^{31}$.

C'est aussi en 2006, suite à l'élection d'un gouvernement conservateur mené par Stephen Harper, que le Québec s'est fait accorder une place au sein de la délégation canadienne à l'Organisation des Nations unies pour l'éducation, la science et la culture (UNESCO) ${ }^{32}$. Cependant, ce gain provincial n'a rien de si surprenant, car la doctrine Gérin-Lajoie, existant

\footnotetext{
${ }^{27}$ P. Resnick. Le Canada (2013), 34.

${ }^{28}$ P. Resnick. Le Canada (2013), 34-45.

${ }^{29}$ M. Bock-Cöté. De la reconnaissance du Québec (2007), 6.

${ }^{30}$ Ibid., 16.

${ }^{31}$ Ibid., 15.

${ }^{32}$ Premier Ministre du Canada/Stephen Harper. Le Premier ministre Harper et le premier ministre Charest signent un accord historique établissant un rôle formel pour le Québec à l'UNESCO (Québec 2006).
} 
depuis 1965, permet aux provinces d'agir à l'international dans leurs champs de compétence ${ }^{33}$. Pour Réjean Pelletier, ce geste représente peu, car la délégation canadienne à l'UNESCO ne devant parler que d'une seule voix, le Québec est donc limité à une fonction de lobbyiste auprès des autres membres de la délégation canadienne ou des autres pays membres, d'où la qualification de strapontin ${ }^{34}$. Ces propos sont à nuancer, car malgré les limites de la participation québécoise à l'UNESCO, celle-ci permet tout de même au Québec d'avoir des relations internationales directes avec des États souverains et des États fédérés au sein de cette organisation internationale ${ }^{35}$.

Rappelons cependant qu'en cas de non consensus au sein de la délégation canadienne, le Québec peut demander des explications formelles de la part d'Ottawa; de plus, il a la liberté de poser des questions lors du suivi des décisions prises $^{36}$. Pour Kymlicka, la motion reconnaissant les Québécois comme une nation et l'attribution d'un siège à l'UNESCO est signe d'un rapprochement vers une forme de fédération multinationale ${ }^{37}$.

Ensuite, comme je l'ai présenté lors de la conceptualisation du fédéralisme, ce type d'organisation des pouvoirs implique une séparation des compétences entre le palier fédéral et les provinces. Le bilan du gouvernement conservateur quant au respect des champs de compétences étudiera de façon détaillé le règlement du déséquilibre fiscal, l'utilisation du pouvoir de dépenser, car ce sont des spécificités de la politique canadienne qui permettent au gouvernement fédéral de s'ingérer dans les compétences provinciales et auxquelles Stephen

\footnotetext{
${ }^{33}$ E. Montpetit. Le fédéralisme d'ouverture (Québec, 2007), 109.

${ }^{34} \mathrm{R}$. Pelletier. Le renouvellement de la fédération canadienne (2013) 42.

${ }^{35}$ E. Montpetit. Le fédéralisme d'ouverture (Québec, 2007), 110.

${ }^{36}$ Y. Charbonneau. Le Québec peut faire valoir sa voix à l'UNESCO (2011).

${ }^{37}$ J-F. Caron et G. Laforest. Canada and Multinational Federalism: From the Spirit of 1982 to Stephen Harper's Open Federalism (2009), 45-46.
} 
Harper avait promis de s'attaquer.

Depuis 2010, le gouvernement fédéral tente de créer une commission pancanadienne des valeurs mobilières, à laquelle six provinces s'opposent, dont le Québec ${ }^{38}$. En s'entêtant à créer une telle commission, le gouvernement de Stephen Harper s'ingèrerait directement dans une compétence provinciale, dont fait partie la régulation des valeurs immobilières ${ }^{39}$.

Lors du budget de mars 2007, Stephen Harper a modifié la formule de péréquation et procédé à une hausse des transferts sociaux aux provinces pour ainsi remédier au problème du déséquilibre fiscal ${ }^{40}$. Après avoir nettement amélioré la situation du Québec et des provinces en général, il est revenu sur sa décision un an plus tard, en raison de conjoncture économique, résultant en une perte de 50 millions de dollars pour le Québec en 2009-2010¹. Le ministre québécois des Finances Raymond Bachand recevait encore moins de fonds issus de transferts fédéraux pour l'année 2010-2011 ${ }^{42}$.

En décembre dernier, Jim Flaherty, ministre des Finances canadien, annonçait que les transferts aux provinces pour les soins de santé, qui augmentaient de $6 \%$ par année, seraient indexés au taux de croissance économique à partir de $2017^{43}$. De plus, le transfert canadien en matière de santé (TCS) sera strictement distribué au prorata de la population, sans tenir compte des spécificités régionales ${ }^{44}$. Pour le Parti conservateur, le déséquilibre fiscal est chose réglé

\footnotetext{
${ }^{38}$ Radio-Canada et La Presse Canadienne. Valeurs mobilières : une commission nationale inconstitutionnelle (2011).

${ }^{39} \mathrm{~J}$. Poirier. Le partage des compétences et les relations intergouvernementales : la situation au Canada dans «Le fédéralisme en Belgique et au Canada» (2009), 112.

${ }^{40}$ A. Noel. Fédéralisme d'ouverture (2008), 27.

${ }^{41}$ A. Castonguay. Péréquation, Ottawa versera moins au Québec l'an prochain (Québec 2008).

${ }^{42}$ L. Godbout. Les cinq années au pouvoir de Stephen Harper (2011).

${ }^{43}$ A. Noël. Le fédéralisme symétrique (2012), 104.

${ }^{44}$ Ibid., 104.
} 
depuis $2007^{45}$. Le gouvernement du Québec et celui de bien d'autres provinces ne partagent cependant pas le même avis ${ }^{46}$.

En 2011, Stephen Harper remettait une enveloppe de 2,2 milliards de dollars au gouvernement québécois de Jean Charest dû à l'harmonisation de la TPS et de la TVQ ${ }^{47}$. Cela représentait, pour le gouvernement québécois de l'époque et le gouvernement canadien, une grande réussite du fédéralisme d'ouverture.

Finalement, l'existence d'un déséquilibre fiscal entre le Fédéral et les provinces permet au gouvernement central de s'ingérer de manière indirecte dans des compétences provinciales, position que tiennent depuis longtemps les différents gouvernements du Québec ${ }^{48}$.

La proposition du gouvernement fédéral s'est trouvé à être de limiter le pouvoir de dépenser dans les compétences provinciales, mais que dans les nouveaux programmes à frais partagés ${ }^{49}$. Ce pouvoir n'étant pas reconnu officiellement par la constitution, un tel accord ferait reconnaître au gouvernement québécois l'existence de ce pouvoir qu'il conteste, entraînant l'enlisement des négociations ${ }^{50}$.

En avril 2013, Alexandre Cloutier, ministre québécois délégué aux affaires intergouvernementales, affirmait que la volonté du gouvernement conservateur d'éliminer le

\footnotetext{
${ }^{45}$ La Presse Canadienne. Paiements de péréquation - Le Québec n'a pas à se plaindre, disent les conservateurs (2009).

${ }^{46}$ Portail Québec. Le ministre Nicolas Marceau fait le point sur la rencontre des ministres des Finances des provinces et des territoires (Toronto 2013).

${ }^{47} \mathrm{M}$. Ouellet. Taxe de vente harmonisée: Harper donne enfin son chèque de 2,2 milliards \$: TVH: Harper donne 2,2 milliards \$ au Québec (Montréal 2011).

${ }^{48}$ H. Lazard. The Spending Power and the Harper Government (2008), 129.

${ }^{49}$ M-A. Ménard. Le pouvoir fédéral de dépenser (2007).

${ }^{50}$ A. Noel. Fédéralisme d'ouverture (2008), 29.
} 
pouvoir de dépenser ne s'était jamais concrétisée ${ }^{51}$.

Après avoir passé en revue les points saillants des relations intergouvernementales entre la province du Québec et le palier fédéral depuis l'arrivée au pouvoir de Stephen Harper, il est possible de conclure que «[...]les conservateurs ont respecté leurs promesses d'ouverture, mais sans répondre pleinement aux réclamations du Québec, surtout en matière de péréquation et d'encadrement du pouvoir fédéral de dépenser ${ }^{52}$.» De plus, la motion reconnaissant les québécois comme formant une nation au sein d'un Canada uni, ainsi que l'accord d'un siège pour le Québec au sein de la délégation canadienne à l'UNESCO, malgré l'inéquivoque démonstration d'ouverture, ne constitue que des gains symboliques pour le Québec ${ }^{53}$. Le Parti conservateur, qui mettait le respect des compétences de juridiction provinciale au centre de leur promesse d'ouverture, l'a clairement bafouée avec la tentative de créer une commission des valeurs mobilières à l'échelle nationale.

Il serait intéressant de se demander si les choix politiques que les Québécois ont fait aux élections fédérales de 2008 et 2011 auraient «[...]contribué à sortir le Québec de l’écran de radar du gouvernement conservateur ${ }^{54} . \gg$ Il est aussi pertinent de rappeler qu'avant même l'adoption de la loi sur la clarté référendaire (C-20), élaborée par Stéphane Dion du Parti libéral du Canada, Stephen Harper avait rédigé, en 1996, un projet de loi délimitant le cadre dans lequel le Québec pourrait accéder à la souveraineté $(\mathrm{C}-341)^{55}$. Ce projet de loi, mort peu après au feuilleton, précédait de peu l'initiative libérale qui a mis sous tutelle canadienne le droit à

\footnotetext{
${ }^{51}$ L. Gagné. Le PQ compte défendre les intérêts du Québec (2013).

${ }^{52} \mathrm{R}$. Pelletier. Le renouvellement de la fédération canadienne (2013), 43.

${ }^{53}$ G. Boismenu. Quand les références de la communication en politique fichent le camp (2013), 73-74.

${ }^{54}$ E. Montpetit. Le fédéralisme d'ouverture (Québec, 2007), 146.

${ }^{55}$ C. Hénert. Stephen Harper : l'ombre et la clarté (2013).
} 
l'autodétermination de la nation québécoise ${ }^{56}$. Son parti est même allé récemment contester la loi québécoise qui n'allait pas dans le même sens que la loi C- $20^{57}$. Il serait donc difficile de conclure que Stephen Harper est parvenu à mettre fin à l'ère de confrontation entre le gouvernement canadien et le Québec.

${ }^{56}$ M. Bock-Cöté. De la reconnaissance du Québec (2007), 15.

${ }^{57}$ Agence QMI. Harper veut invalider la loi 99 (2013). 


\section{Bibliographie}

Agence QMI. « Harper veut invalider la loi 99 », Le Journal de Montréal, [En ligne], 19 octobre 2013, http://www.journaldemontreal.com/2013/10/19/harper-veut-invalider-laloi-99, (Page consultée le 25 novembre 2013)

Bock-Cöte, Mathieu. «De la reconnaissance du Québec à celle des Québécois: retour sur la reconnaissance de la nation par le gouvernement de Stephen Harper à l'automne 2006 », Institut de recherche sur le Québec, [En ligne], Avril 2007, pp.1-18. http://irq.qc.ca/storage/etudes/IRQ-Etude_MBC_final_8.pdf, (Page consultée le 20 octobre 2013).

Caron, Jean-François et Guy, LAFOREST. «Canada and Multinational Federalism: From the Spirit of 1982 to Stephen Harper's Open Federalism », Nationalism and Ethnic Politics, Vol.15, No.1, 2009, pp.27-55.

Castonquay, Alec. «Péréquation, Ottawa versera moins au Québec l'an prochain », Le Devoir, Québec, 4 novembre 2008, http://www.ledevoir.com/politique/quebec/214066/perequation-ottawa-versera-moins-auquebec-l-an-prochain, (Page consultée le 29 novembre 2013).

Couture, Claude et David Chandonnet. Vingt ans après Charlottetown : L'ordre libéral au Canada, Québec, Les Presses de l'Université Laval, 2013, 93 p.

Dutrisac, Robert et La Presse Canadienne. «Le Québec reconnu comme nation-Une motion sans conséquence, avoue Ottawa », Le Devoir, [En ligne], Canada, 24 novembre 2006, http://www.ledevoir.com/politique/canada/123493/le-quebec-reconnu-comme-nationune-motion-sans-consequence-avoue-ottawa, (Page consultée le 25 novembre 2013).

Équipe Perspective Monde, «Définition de Fédération dans le glossaire », [En ligne], septembre 2013, http://perspective.usherbrooke.ca/bilan/servlet/BMDictionnaire?iddictionnaire=1500, (Page consultée le 26 octobre 2013).

Gagné, Louis. « Assurance-emploi : Le PQ compte défendre les intérêts du Québec », Agence $Q M I, 3$ avril 2013, [En ligne], http://tvanouvelles.ca/lcn/infos/national/archives/2013/04/20130403-103945.html (Page consultée le 22 septembre 2013). 
Godbout, Luc. «Les cinq années au pouvoir de Stephen Harper », La Tribune, [En ligne], 17 janvier 2011, http://www.lapresse.ca/la-tribune/opinions/201101/17/01-4360837-lescinq-annees-au-pouvoir-du-gouvernement-harper.php, (Page consultée le 2 décembre 2013)

Hamel, Pierre et Jean-Michel Lacroix (dir), « Les relations Québec-Canada : arrêter le dialogue de sourds ? », Peter Lang, Bruxelles, 2013, 150 p.

Harmes, Adams. «The Political Economy of Open Federalism », Canadian Journal of Political Science, Vol.40, No.2, Juin 2007, pp.417-437.

Hébert, Chantal. «Stephen Harper : l'ombre et la clarté », L'actualité, [En ligne], 26 février 2013, http://www.lactualite.com/actualites/chronique-de-chantal-hebert/stephen-harperlombre-et-la-clarte/, (Page consultée le 20 mars 2014).

Laforest, Guy. «The $20^{\text {th }}$ annual Mcdonald lecture in constitutional studies: What Canadian Federalism Means in Québec ». Review Of Constitutional Studies, Vol.15, No.1, 2010, pp. 1-33.

La Presses Canadienne. «Paiements de péréquation - Le Québec n'a pas à se plaindre, disent les conservateurs », Le Devoir, 22 janvier 2009, http://www.ledevoir.com/politique/canada/228703/paiements-de-perequation-le-quebecn-a-pas-a-se-plaindre-disent-les-conservateurs, (Page consultée le $1^{\text {er }}$ décembre 2013).

Lazard, Harvey. «The Spending Power and the Harper Government », Queen's Law Journal, Vol.34, No.1, automne 2008, pp.125-140.

Ménard, Marc-Antoine. «Le pouvoir fédéral de dépenser », Radio-Canada, 23 octobre 2007, http://www.radio-canada.ca/nouvelles/National/2007/10/22/002-Pouvoir-federal.shtml, (Page consultée le 2 décembre 2013).

Monière, Denis. Pour comprendre le nationalisme au Québec et ailleurs, Québec, Les Presses de l'Université de Montréal, 2001, 148p.

Montpetit, Éric. Le fédéralisme d'ouverture. La recherche d'une légitimité canadienne au Québec, Sillery, Septentrion, 2007, 138 p.

Noël, Alain. «Fédéralisme d'ouverture et pouvoir de dépenser au Canada », Revista d'Estudis Autonòmics i Federals, Barcelone, Vol. 7, No.1, octobre 2008, pp. 10-36. 
Noël, Alain. «Le fédéralisme symétrique », Option politiques, février 2012, p.104.

Ouellet, Marin. «Taxe de vente harmonisee: Harper donne enfin son chèque de 2,2 milliards \$: TVH: Harper donne 2,2 milliards \$ au Quebec », La presse canadienne, Montréal, [En ligne], 30 septembre 2011, http://www.lapresse.ca/actualites/politique/politiquecanadienne/201109/30/01-4452988-tvh-ottawa-remet-22-milliards-au-quebec.php, (Page consultée le $1^{\text {er }}$ novembre 2013).

Pelletier, Réjean et Manon, Tremblay (dir), Le parlementarisme canadien, 4 édition, Québec, Les Presses de l'Université Laval, 2009, 581 p.

Pelletier, Réjean. Le Québec et le fédéralisme canadien. Un regard critique, Québec, Les Presses de l'Université Laval, 2008, 236p.

Poirier, Johanne. Le partage des compétences et les relations intergouvernementales : la situation au Canada dans «Le fédéralisme en Belgique et au Canada», De Boeck Supérieur, 2009, pp.107-122.

Portail Québec, «Le ministre Nicolas Marceau fait le point sur la rencontre des ministres des Finances des provinces et des territoires », Actualités gouvernementales, Toronto, $1^{\mathrm{er}}$ novembre 2013, http://communiques.gouv.qc.ca/gouvqc/communiques/GPQF/Novembre2013/01/c9487.h $\underline{\text { tml, }}$ (Page consultée le $1^{\text {er }}$ décembre 2013).

Premier Ministre Du Canada/Stephen Harper. Le Premier ministre Harper et le premier ministre Charest signent un accord historique établissant un rôle formel pour le Québec à l'UNESCO, [En ligne], Québec, 5mai 2006, http://pm.gc.ca/fra/nouvelles/2006/05/05/premier-ministre-harper-et-premier-ministrecharest-signent-accord-historique, (Page consultée le 5 décembre 2013).

Secrétariat aux Affaires Intergouvernementales Canadiennes, Legouvernement Marois s'attend à ce que le gouvernement Harper rectifie le tir dans le domaine économique : communiqué de presse du 10 octobre 2013, Québec, 10 octobre 2013, [En ligne], http://www.saic.gouv.qc.ca/centre_de_presse/communiques/2013/2013-10-10.html, (Page consultée le $1^{\text {er }}$ novembre 2013). 